ARTICLE

\title{
Magnetic field reveals vanishing Hall response in the normal state of stripe-ordered cuprates
}

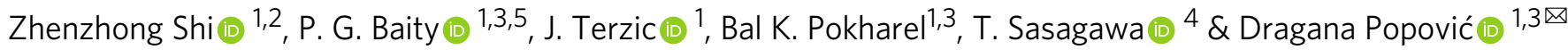

The origin of the weak insulating behavior of the resistivity, i.e. $\rho_{x x} \propto \ln (1 / T)$, revealed when magnetic fields $(H)$ suppress superconductivity in underdoped cuprates has been a longtime mystery. Surprisingly, the high-field behavior of the resistivity observed recently in chargeand spin-stripe-ordered La-214 cuprates suggests a metallic, as opposed to insulating, highfield normal state. Here we report the vanishing of the Hall coefficient in this field-revealed normal state for all $T<(2-6) T_{\mathrm{c}^{\prime}}^{0}$ where $T_{\mathrm{c}}^{0}$ is the zero-field superconducting transition temperature. Our measurements demonstrate that this is a robust fundamental property of the normal state of cuprates with intertwined orders, exhibited in the previously unexplored regime of $T$ and $H$. The behavior of the high-field Hall coefficient is fundamentally different from that in other cuprates such as $\mathrm{YBa}_{2} \mathrm{Cu}_{3} \mathrm{O}_{6+x}$ and $\mathrm{YBa}_{2} \mathrm{Cu}_{4} \mathrm{O}_{8}$, and may imply an approximate particle-hole symmetry that is unique to stripe-ordered cuprates. Our results highlight the important role of the competing orders in determining the normal state of cuprates.

\footnotetext{
${ }^{1}$ National High Magnetic Field Laboratory, Florida State University, Tallahassee, FL 32310, USA. ${ }^{2}$ School of Physical Science and Technology \& Institute for Advanced Study, Soochow University, Suzhou 215006, China. ${ }^{3}$ Department of Physics, Florida State University, Tallahassee, FL 32306, USA. ${ }^{4}$ Materials and Structures Laboratory, Tokyo Institute of Technology, Kanagawa 226-8503, Japan. ${ }^{5}$ Present address: James Watt School of Engineering, University of Glasgow, Glasgow G12 8QQ, Scotland, United Kingdom. ${ }^{凶}$ email: dragana@magnet.fsu.edu
} 
T he central issue for understanding the high-temperature superconductivity in cuprates is the nature of the ground state that would have appeared had superconductivity not intervened. Therefore, magnetic fields have been commonly used to suppress superconductivity and expose the properties of the normal state, but the nature of the high- $H$ normal state may be further complicated by the interplay of charge and spin orders with superconductivity. $\mathrm{La}_{2-x-y} \mathrm{Sr}_{x}(\mathrm{Nd}, \mathrm{Eu})_{y} \mathrm{CuO}_{4}$ compounds are ideal candidates for probing the nature of the field-revealed ground state ${ }^{1}$ of underdoped cuprates in the presence of intertwined orders because, for doping levels near $x=1 / 8$, they exhibit both spin and charge orders with the strongest correlations and lowest $T_{c}^{0}$ already at $H=0$. In particular, in each $\mathrm{CuO}_{2}$ plane, charge order appears in the form of static stripes that are separated by charge-poor regions of oppositely phased antiferromagnetism ${ }^{2}$, i.e. spin stripes, with the onset temperatures $T_{\mathrm{CO}}>T_{\mathrm{SO}}>T_{\mathrm{c}}^{0}$; stripes are rotated by $90^{\circ}$ from one layer to next. The low values of $T_{\mathrm{c}}^{0}$ have made it possible to determine the in-plane $T-H$ vortex phase diagram ${ }^{3}$ using both linear and nonlinear transport over the relatively largest range of $T$ and perpendicular $H$ (i.e., $H \perp \mathrm{CuO}_{2}$ layers), and to probe deep into the high-field normal state. The most intriguing question, indeed, is what happens after the superconductivity is suppressed by $H$, i.e. for fields greater than the quantum melting field of the vortex solid where $T_{\mathrm{c}}(H) \rightarrow 0$. It turns out that a wide regime of vortex liquid-like behavior, i.e. strong superconducting (SC) phase fluctuations, persists in twodimensional (2D) $\mathrm{CuO}_{2}$ layers, all the way up to the upper critical field $H_{\mathrm{c} 2}$. It is in this regime that recent electrical transport measurements have also revealed ${ }^{4}$ the signatures of a spatially modulated SC state referred to as a pair density wave ${ }^{5}$ (PDW). The normal state, found at $H>H_{\mathrm{c} 2}$, is highly anomalous ${ }^{3}$ : it is characterized by a weak, insulating $T$-dependence of the in-plane longitudinal resistivity, $\rho_{x x} \propto \ln (1 / T)$, without any sign of saturation down to at least $T / T_{\mathrm{c}}^{0} \sim 10^{-2}$, and the negative magnetoresistance (MR). In contrast to the $H$-independent $\ln (1 / T)$ reported $^{6,7}$ for the case where there is no clear evidence of charge $\operatorname{order}^{8}$ in $H=0$ and where the high- $H$ normal state appears to be an insulator ${ }^{6,9}$, here the $\ln (1 / T)$ behavior is suppressed by $H$, strongly suggesting that $\rho_{x x}$ becomes independent of $T$, i.e., metallic, at high enough magnetic field $(H>70 \mathrm{~T})$. In either case, the origin of such a weak, insulating behavior is not understood $^{7,10-13}$, but it is clear that the presence of stripes seems to affect the nature of the normal state. Therefore, additional experiments are needed to probe the highest- $H$ regime.

In cuprates, the Hall effect has been a powerful probe of the $T=0$ field-revealed normal state (e.g. refs. ${ }^{14-19}$ and refs. therein). In the high-field limit as $T \rightarrow 0$, the Hall coefficient $R_{\mathrm{H}}$, obtained from the Hall resistivity $\rho_{y x}(H)=R_{\mathrm{H}} H$, can be used to determine the sign and the density $(n)$ of charge carriers. In a single-band metal, for example, $n=n_{\mathrm{H}}$, where the Hall number $n_{\mathrm{H}}=1 /\left(e R_{\mathrm{H}}\right)$ and $e$ is the electron charge $\left(R_{\mathrm{H}}>0\right.$ for holes, $R_{\mathrm{H}}<0$ for electrons). In general, the magnitude of $R_{\mathrm{H}}$ reflects the degree of particlehole asymmetry and, thus, understanding the Hall coefficient provides deep insight into the microscopic properties. However, the interpretation of the Hall effect in cuprates has been a challenge, because $R_{\mathrm{H}}$ can depend on both $T$ and $H$, and it can be affected by various factors, such as the presence of SC correlations and the topological structure of the Fermi surface. For example, a drop of $R_{\mathrm{H}}$ from positive to negative values with decreasing $T$, observed in underdoped cuprates for dopings where charge orders are present ${ }^{20}$ at high $H$, has been attributed ${ }^{14,19,21,22}$ to the Fermi surface reconstruction, which includes the appearance of electron pockets in the Fermi surface of a hole-doped cuprate. A drop in the normal state, positive $R_{\mathrm{H}}(T)$ is, in fact, observed in all hole-doped cuprates near $x=1 / 8$ (see ref. ${ }^{21}$ and refs. therein). Other studies of the Hall effect in cuprates have focused on the effects of SC fluctuations (refs. ${ }^{23,24}$ and refs. therein), and on the pronounced change in the Hall number across the charge order and the pseudogap quantum critical points ${ }^{16-18,25,26}$. However, the Hall behavior in the $T \rightarrow 0, H>H_{\mathrm{c} 2}$ regime has remained mostly unexplored. In particular, recent studies of the $\mathrm{La}_{2-x-y}$ $\mathrm{Sr}_{x}(\mathrm{Nd}, \mathrm{Eu})_{y} \mathrm{CuO}_{4}$ compounds have demonstrated ${ }^{3,4}$ that reliable extrapolations to the $T \rightarrow 0$ normal state can be made only by tracking the evolution of SC correlations down to $T \ll T_{\mathrm{c}}^{0}$ and $H / T_{\mathrm{c}}^{0}[\mathrm{~T} / \mathrm{K}] \gg 1$, but there have been no studies of the Hall effect in stripe-ordered cuprates that extend to that regime of $T$ and $H$ and, specifically, to the anomalous normal state at $H>H_{\mathrm{c} 2}$.

Therefore, we measure the Hall effect on $\mathrm{La}_{1.7} \mathrm{Eu}_{0.2} \mathrm{Sr}_{0.1} \mathrm{CuO}_{4}$ and $\mathrm{La}_{1.48} \mathrm{Nd}_{0.4} \mathrm{Sr}_{0.12} \mathrm{CuO}_{4}$ (see Methods) over the entire in-plane $T-H$ vortex phase diagram previously established ${ }^{3,4}$ for $T$ down to $T / T_{\mathrm{c}}^{0} \lesssim 0.003$ and fields up to $H / T_{\mathrm{c}}^{0} \sim 10 \mathrm{~T} / \mathrm{K}$, and deep into the normal state. Combining the results of several techniques allows us to achieve an unambiguous interpretation of the Hall data for $H<H_{\mathrm{c} 2}$, and reveal novel properties of the normal state for $H>H_{\mathrm{c} 2}$. Our main results are summarized in the $T-H$ phase diagrams shown in Fig. 1. The key finding is that, in the high-field limit, the positive $R_{\mathrm{H}}$ decreases to zero at $T=T_{0}(H)$ upon cooling, and it remains zero (see Methods) all the way down to the lowest measured $T$, despite the absence of any observable signs of superconductivity. Here, $T_{0}(H) \sim(2-3) T_{\mathrm{c}}^{0}$ for $\mathrm{La}_{1.7} \mathrm{Eu}_{0.2} \mathrm{Sr}_{0.1^{-}}$ $\mathrm{CuO}_{4}$ and $T_{0}(H) \sim 6 T_{\mathrm{c}}^{0}$ for $\mathrm{La}_{1.48} \mathrm{Nd}_{0.4} \mathrm{Sr}_{0.12} \mathrm{CuO}_{4}$. $T_{\mathrm{c}}^{0}$, where the linear resistivity $\rho_{x x}$ becomes zero, and other characteristic temperatures, such as the pseudogap $T_{\mathrm{PG}}$, are summarized in Table 1. Therefore, the vanishing Hall coefficient appears well below $T_{\mathrm{PG}}$, in the temperature region where both charge and spin orders (i.e., stripes) have fully developed. Meanwhile, we note that the drop of $R_{\mathrm{H}}$ at $T>T_{0}$ does not depend on $H$, while $T_{0}(H)$ is very weakly dependent on $H$ (Fig. 1), almost constant, suggesting that $R_{\mathrm{H}} \approx 0$ is characteristic of the zero-field (normal) ground state in the presence of stripes.

\section{Results}

Hall coefficient. Our main results are shown in Fig. 1. From the Hall measurements, we are able to identify regions in $(T, H)$ phase space with different signs of $R_{\mathrm{H}}$ (Fig. 1a, b) and, in particular, we find $R_{\mathrm{H}} \approx$ 0 over a wide range of $T$ and $H$ in both materials. Further insight is obtained by comparing the Hall results with the phase diagram obtained by other transport techniques, as shown in Fig. 1c, d. The measurements of the in-plane magnetoresistance $\rho_{x x}(H)$ at different $T$ were used to determine ${ }^{3,4} T_{\mathrm{c}}(H)$, the melting temperature of the vortex solid in which $\rho_{x x}=0$. Although the quantum melting fields of the vortex solid are relatively low $(\sim 5.5 \mathrm{~T}$ and $\sim 4 \mathrm{~T}$, respectively, for $\mathrm{La}_{1.7} \mathrm{Eu}_{0.2} \mathrm{Sr}_{0.1} \mathrm{CuO}_{4}$ and $\left.\mathrm{La}_{1.48} \mathrm{Nd}_{0.4} \mathrm{Sr}_{0.12} \mathrm{CuO}_{4}\right)$, the regime of strong 2D SC phase fluctuations (vortex liquid) extends up to much higher fields ${ }^{3,4} H_{\text {peak }}(T) \sim H_{\mathrm{c} 2}(T)$, where $H_{\text {peak }}(T)$ is the position of the peak in $\rho_{x x}(H)$. For $T \rightarrow 0, H_{\mathrm{c} 2} \sim 20 \mathrm{~T}$ for $\mathrm{La}_{1.7} \mathrm{Eu}_{0.2} \mathrm{Sr}_{0.1} \mathrm{CuO}_{4}$ and $H_{\mathrm{c} 2} \sim 25 \mathrm{~T}$ for $\mathrm{La}_{1.48} \mathrm{Nd}_{0.4} \mathrm{Sr}_{0.12} \mathrm{CuO}_{4}$.

Figure 2 shows the field dependence of $R_{\mathrm{H}}=\rho_{y x}(H) / H$ for various $T$ in both materials (see Supplementary Figs. 1 and 2 for the $\rho_{y x}(H)$ data at different $\left.T\right)$. At relatively high $T>T_{0}>T_{c}^{0}$ in the pseudogap regime, the positive $R_{\mathrm{H}}$ is independent of $H$ (Fig. 2a, c), as observed in conventional metals, although the in-plane transport is already insulatinglike, i.e. $d \rho_{x x} / d T<0$ (Fig. 3a, c, also Supplementary Fig. 5). Upon cooling, $R_{\mathrm{H}}$ decreases to zero at $T=T_{0}(H)$, and then becomes negative in the regime of lower fields. The field dependence remains weak at all $T$, similar to the observations ${ }^{27}$ in striped $\mathrm{La}_{1.905} \mathrm{Ba}_{0.095} \mathrm{CuO}_{4}$, but in contrast to the strong $H$-dependence of $R_{\mathrm{H}}$ in $\mathrm{YBa}_{2} \mathrm{Cu}_{3} \mathrm{O}_{6+x}$ and $\mathrm{YBa}_{2} \mathrm{Cu}_{4} \mathrm{O}_{8}\left(\mathrm{YBCO}\right.$ materials; ref. $\left.{ }^{14}\right)$, i.e., in the absence of spin order, or in $\mathrm{La}_{2-x} \mathrm{Sr}_{x} \mathrm{CuO}_{4}$ (ref. ${ }^{25}$ ), where the charge order is at best very weak. The most striking finding is that, at the 

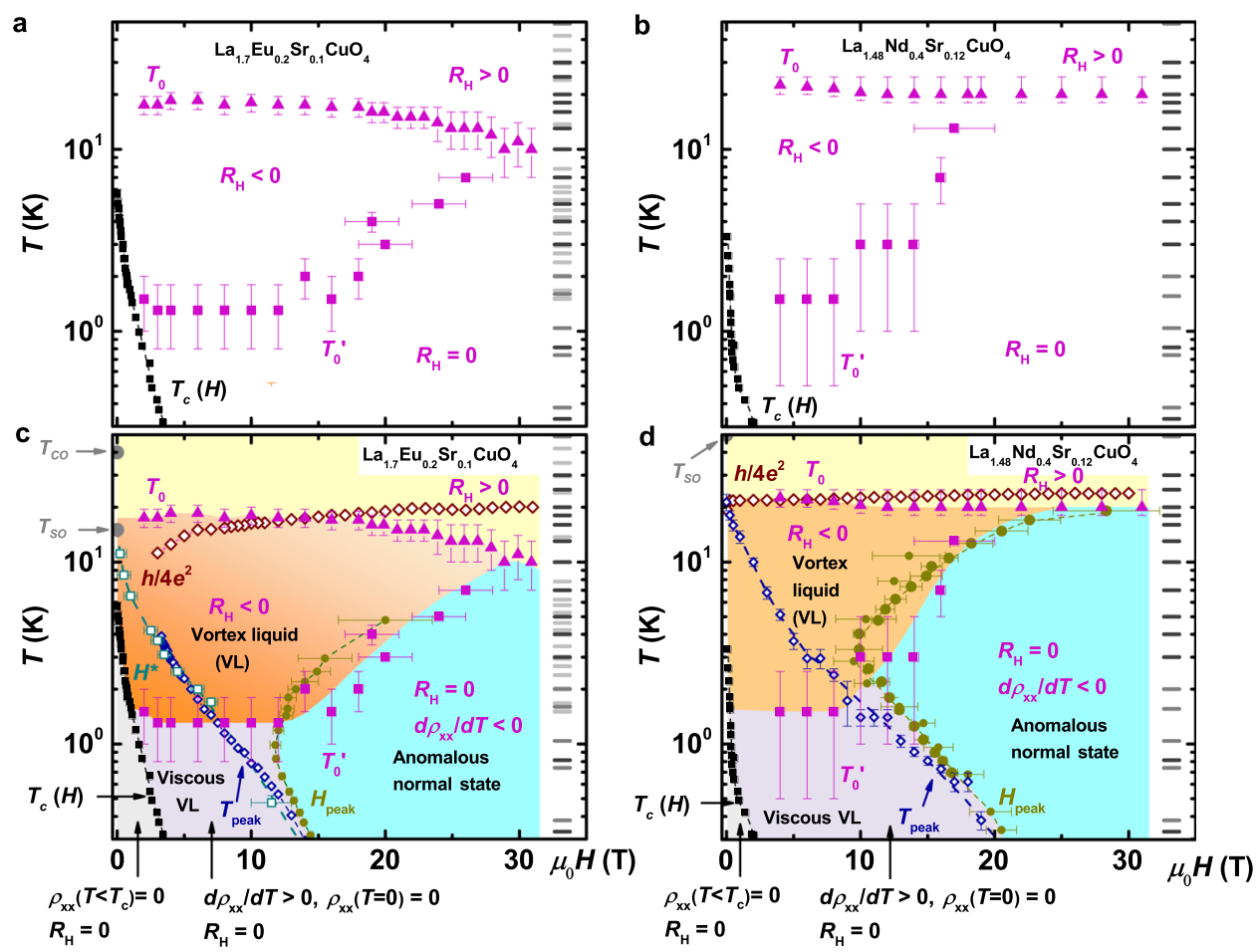

Fig. 1 In-plane Hall coefficient $\boldsymbol{R}_{\mathbf{H}}$ across the $\boldsymbol{T}$-H phase diagram of striped cuprates. a, b Regions of $T$ and $H$ with different signs of $R_{H}$ for $\mathrm{La}_{1.7} \mathrm{Eu}_{0.2} \mathrm{Sr}_{0.1} \mathrm{CuO}_{4}$ and $\mathrm{La}_{1.48} \mathrm{Nd}_{0.4} \mathrm{Sr}_{0.12} \mathrm{CuO}_{4}$, respectively. c, d Comparison of the results for $R_{\mathrm{H}}$ to the other transport data ${ }^{3,4}$ for $\mathrm{La}_{1.7} \mathrm{Eu}_{0.2} \mathrm{Sr}_{0.1} \mathrm{CuO}_{4}$ and $\mathrm{La}_{1.48} \mathrm{Nd}_{0.4} \mathrm{Sr}_{0.12} \mathrm{CuO}_{4}$, respectively. $T_{\mathrm{c}}(H)$ (black squares): boundary of the vortex solid in which $\rho_{x x}\left(T<T_{\mathrm{c}}\right)=0$ and $R_{\mathrm{H}}=0$, as expected for a superconductor. The upper critical field $H_{\mathrm{c} 2}(T) \sim H_{\text {peak }}(T) ; H_{\text {peak }}(T)$ (dark green dots) are the fields above which the magnetoresistance changes from positive to negative ${ }^{3,4}$. The low- $T$, viscous vortex liquid $(V L)$ regime (light violet) is bounded by $T_{c}(H)$ and, approximately, by $T_{\text {peak }}(H)$ (positions of the peak in $\rho_{x x}(T)$; open blue diamonds), $H^{*}(T)$ (crossover between non-Ohmic and Ohmic behavior ${ }^{3}$; open royal squares), or $H_{\text {peak }}(T)$; here the behavior is metallic $\left(d \rho_{x x} / d T>0\right)$ with $\rho_{x x}(T \rightarrow 0)=0$ and $R_{H}=0$. The field-revealed normal state (blue) exhibits anomalous behavior: $\rho_{x x}(T)$ has an insulating, In $(1 / T)$ dependence 3,4 , but $R_{H}=0$ despite the absence of superconductivity. At high $T$ (yellow), $R_{H}>0$ and drops to zero at $T=T_{0}(H)$ (magenta triangles). In the high- $T$ VL regime $\left(H<H_{\text {peaki }}\right.$ dark beige), $R_{H}$ becomes negative before vanishing at lower $T=T_{0}^{\prime}(H)$ (magenta squares), as the vortices become less mobile. The $h / 4 e^{2}$ symbols (open brown diamonds) show the $(T, H)$ values where the sheet resistance changes from $R_{\square / l a y e r}<R_{Q}=h / 4 e^{2}$ at higher $T$, to $R_{\square \text { /layer }}>R_{\mathrm{Q}}$ at lower $T$. Zero-field values of $T_{\mathrm{SO}}$ and $T_{\mathrm{CO}}$ are also shown; $T_{\mathrm{PG}} \sim 175 \mathrm{~K}$ and $150 \mathrm{~K}$ for $\mathrm{La}_{1.7} \mathrm{Eu}_{0.2} \mathrm{Sr}_{0.1} \mathrm{CuO}_{4}$ and $\mathrm{La}_{1.48} \mathrm{Nd}_{0.4} \mathrm{Sr}_{0.12} \mathrm{CuO}_{4}$, respectively ${ }^{55}$. All dashed lines guide the eye. In all panels, gray horizontal marks indicate measurement temperatures in different runs, the resolution of which defines vertical error bars for $T_{0}$ and $T_{0}^{\prime}$; horizontal error bars reflect the uncertainty in defining $T_{0}^{\prime}$ within our experimental resolution (see Supplementary Fig. 3 for the raw $R_{\mathrm{H}}(H)$ data).

Table 1 Characteristic temperatures.

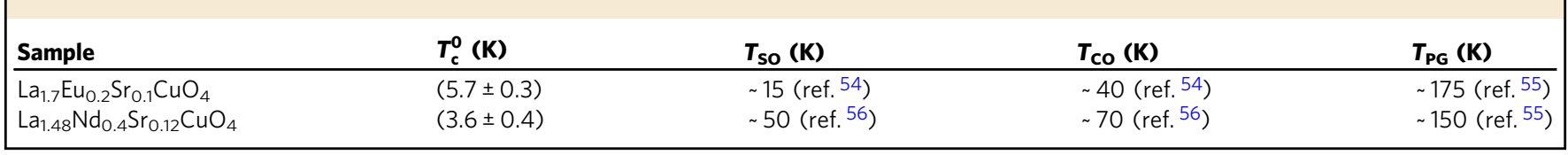

highest fields $\left(H>H_{\text {peak }} \sim H_{\mathrm{c} 2}\right), R_{\mathrm{H}}$ remains immeasurably small for $T<T_{0}$, down to the lowest measured $T$ (Fig. 2b, d). In other words, for a fixed $T<T_{0}(H), R_{\mathrm{H}}<0$ at low $H$, but it becomes zero and remains zero (see Methods) with increasing field.

In Fig. 3, we compare $R_{\mathrm{H}}(T)$ and $\rho_{x x}(T)$ for various fields. The drop of $R_{\mathrm{H}}$ observed at $T>T_{0}$ does not depend on $H$ (Fig. 3b, d), similar to earlier studies of the striped La-214 family ${ }^{27-31}$ and other cuprates ${ }^{21}$. The independence of the drop of $R_{\mathrm{H}}$ on field implies that this is a property of the zero-field state, as opposed to some field-induced phase. In YBCO, the drop in $R_{\mathrm{H}}$ was attributed $^{18,21}$ to the Fermi surface reconstruction by charge order. In striped cuprates, however, the onset of the drop in $R_{\mathrm{H}}$ seems closer to the structural phase transition temperature $T_{\mathrm{d} 2}$ (Fig. 3), where $T_{\mathrm{SO}}<T_{\mathrm{CO}}<T_{\mathrm{d} 2}<T_{\mathrm{PG}}$ (ref. ${ }^{3}$ ), but its origin is still under debate ${ }^{27-31}$. We define $T_{0}(H)$ as the temperature at which $R_{\mathrm{H}}$ becomes zero or negative, and it is apparent that it has a very weak, almost negligible field dependence. $T_{0}\left[\sim(2-3) T_{c}^{0}\right.$ for $\mathrm{La}_{1.7} \mathrm{Eu}_{0.2} \mathrm{Sr}_{0.1} \mathrm{CuO}_{4}$; $\sim 6 T_{\mathrm{c}}^{0}$ for $\left.\mathrm{La}_{1.48} \mathrm{Nd}_{0.4} \mathrm{Sr}_{0.12} \mathrm{CuO}_{4}\right]$ is comparable to the temperature at which $\rho_{x x}(T)$ curves in both materials split into either metalliclike (i.e. SClike) or insulatinglike, a correlation that seems to be manifested only in the presence of stripes $^{31}$. We find that, interestingly, this occurs (Fig. $3 \mathrm{a}, \mathrm{c}$ ) when the normal state sheet resistance $R_{\square / \text { layer }} \approx R_{\mathrm{Q}}$, where $R_{\mathrm{Q}}=h /(2 e)^{2}$ is the quantum resistance for Cooper pairs.

Transport in the high- $\boldsymbol{T}, \boldsymbol{H}<\boldsymbol{H}_{\mathrm{c} 2}$ regime. Previous studies have identified $^{3,4}$ the $H<H_{\text {peak }}$ regime as the vortex liquid. The Hall resistivity due to mobile vortex cores is expected ${ }^{32,33}$ to obey the relation $\rho_{x x}{ }^{2} / \rho_{y x} \propto H$, which is indeed observed in this regime in our samples (Supplementary Fig. 6), thus confirming its identification as the vortex liquid. We also find that, in this field range, 

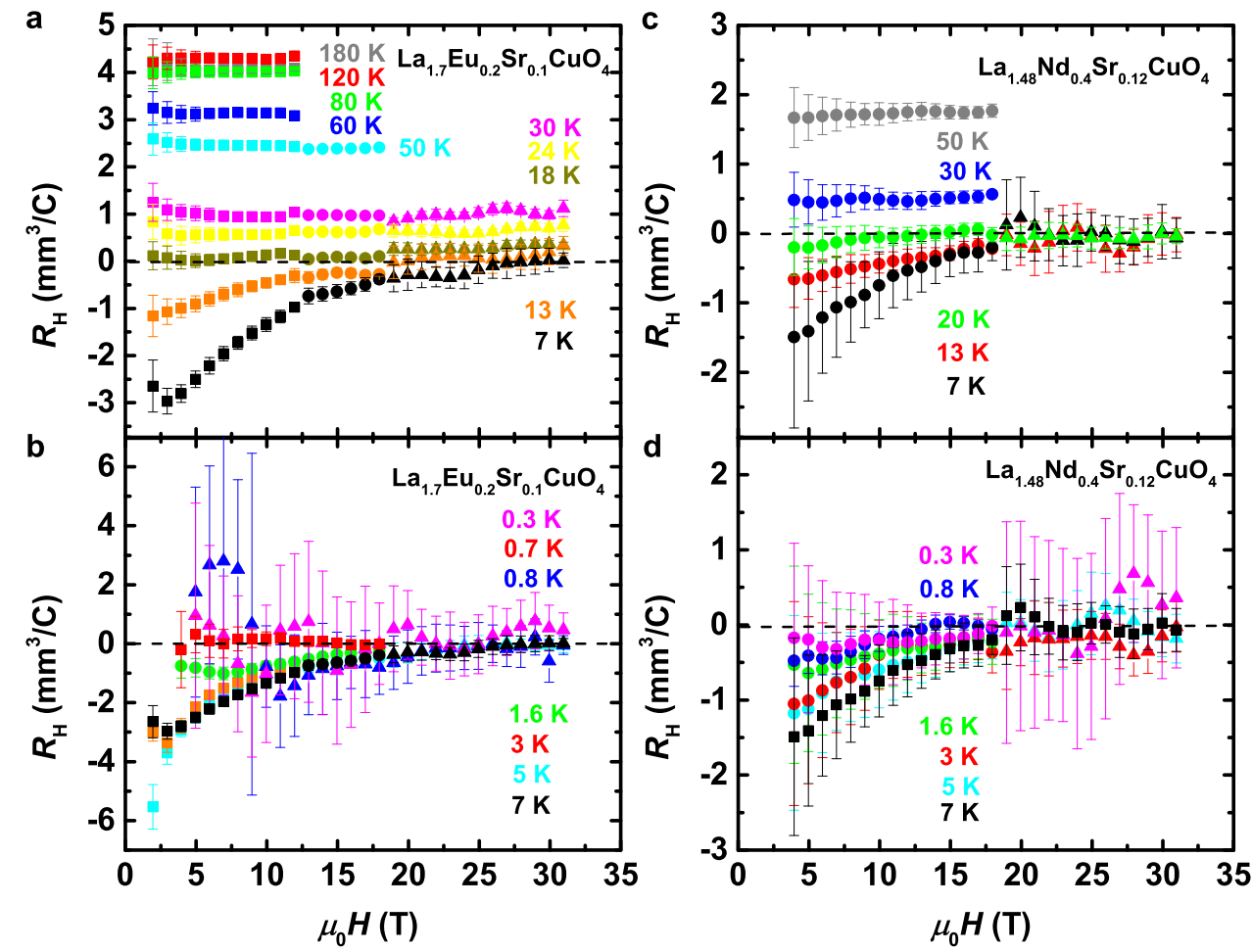

Fig. 2 Field dependence of the Hall coefficient $\boldsymbol{R}_{\mathbf{H}}$ at various temperatures. Higher- and lower- $T$ data for $\mathrm{La}_{1.7} \mathrm{Eu}_{0.2} \mathrm{Sr}_{0.1} \mathrm{CuO}_{4}$ are shown in a and $\mathbf{b}$, respectively, i.e., in $\mathbf{c}$ and $\mathbf{d}$ for $\mathrm{La}_{1.48} \mathrm{Nd}_{0.4} \mathrm{Sr}_{0.12} \mathrm{CuO}_{4}$. Different symbols, corresponding to the data taken in different magnet systems, show good agreement between the runs. The data points represent $R_{\mathrm{H}}$ values averaged over $1 \mathrm{~T}$ bins (Supplementary Fig. 3 ), while error bars correspond to $\pm 1 \mathrm{SD}$ (standard deviation) of the data points within each bin. The error bars are typically larger at lower $T$ resulting from the use of lower excitation currents $I$ (see Methods) necessary to avoid heating and to ensure that the measurements are taken in the $I \rightarrow 0$ limit, because of the strongly nonlinear (i.e., nonOhmic) transport in the presence of vortices ${ }^{3}$. At higher $T$, the error bars are 3-4 times smaller, $\Delta R_{\mathrm{H}} \sim 0.2-0.3 \mathrm{~mm}^{3} / \mathrm{C}$ (see also Supplementary Fig. 3). However, similar $\Delta R_{\mathrm{H}}$, and even $\Delta R_{\mathrm{H}} \sim 0.05 \mathrm{~mm}^{3} / \mathrm{C}$, have been achieved also at low $T$, as described in Methods (see also Supplementary Fig. 4). At high $T$, $R_{\mathrm{H}}$ is independent of $H$, but it decreases to zero at $T=T_{0}(H)$ upon cooling. As $T$ is reduced further, $R_{\mathrm{H}}$ becomes negative for lower $H$, within the $V L$ regime $\left[H<H_{\mathrm{c} 2}(T)\right]$. In the normal state $\left[H>H_{\mathrm{c} 2}(T)\right]$, however, $R_{\mathrm{H}} \approx 0$ down to the lowest $T ; \Delta R_{\mathrm{H}} \sim 0.05 \mathrm{~mm}^{3} / \mathrm{C}$.

$R_{\mathrm{H}}$ is negative for $T_{0}^{\prime}<T<T_{0}$ (dark beige areas in Fig. 1c, d) and it exhibits a minimum, which is suppressed by increasing $H$ (Fig. 3b, d). Such behavior is generally understood ${ }^{14,30,31}$ to result from the vortex contribution to $\rho_{y x}$. The minimum is less pronounced for $x \approx 1 / 8$ (Fig. 3d) than for $x=0.10$ (Fig. 3b), consistent with prior observations ${ }^{30,31}$, as well as with the recent evidence $^{4}$ of a more robust SC PDW state at $x \approx 1 / 8$.

Therefore, the agreement of the results of different techniques allows an unambiguous interpretation of the negative $R_{\mathrm{H}}$ as being dominated by the motion of vortices, even if other effects might, in principle, also contribute to $R_{\mathrm{H}}$. For example, in contrast to stripe-ordered La-214, in $\mathrm{YBa}_{2} \mathrm{Cu}_{3} \mathrm{O}_{y}$ the negative $R_{\mathrm{H}}$ increases with increasing $H$ (ref. ${ }^{14}$ ), suggesting that other effects dominate over the vortex contribution. Our results, however, show that the observation of a field-independent $T_{0}$, at which $R_{\mathrm{H}}$ changes sign, does not necessarily imply that $R_{\mathrm{H}}<0$ is not caused by vortices.

Transport in the low- $\boldsymbol{T}, \boldsymbol{H}<\boldsymbol{H}_{\mathrm{c} 2}$ regime. Similarly, at lower temperatures for $H<H_{\text {peak }}$, in the viscous VL region ${ }^{3}$, the negative $R_{\mathrm{H}}$ is suppressed by decreasing $T$, resulting in $R_{\mathrm{H}}=0$ at $T<T_{0}^{\prime}$ (light violet area in Fig. 1c, d) down to the lowest measured $T=0.019 \mathrm{~K}$ (Fig. $3 \mathrm{~b}, \mathrm{~d}$ insets). Here, $R_{\mathrm{H}}=0$ is thus attributed to the slowing down and freezing of the vortex motion with decreasing $T$ in the presence of disorder. This observation is reminiscent of the zero Hall resistivity observed within the VL regime $\left(H<H_{\mathrm{c} 2}\right)$ in some conventional disordered 2D superconductors $^{34,35}$ and oxide interfaces ${ }^{36}$. Indeed, it has been proposed $^{33}$ that the vanishing of $R_{\mathrm{H}}$ in such so-called "failed superconductors" can be also explained by the strong pinning of the vortex motion.

Incidentally, our results (Fig. 1 and Supplementary Fig. 5) clarify that the origin of $R_{\mathrm{H}}=0$ observed earlier ${ }^{30}$ in $\mathrm{La}_{1.48} \mathrm{Nd}_{0.4}$ $\mathrm{Sr}_{0.12} \mathrm{CuO}_{4}$ for $T \lesssim 5 \mathrm{~K}$ at $9 \mathrm{~T}$ is due to the onset of freezing of the vortex motion. Recently, $R_{\mathrm{H}}=0$ was reported ${ }^{37}$ also in $\mathrm{La}_{2-x}$ $\mathrm{Ba}_{x} \mathrm{CuO}_{4}$ with $x=1 / 8$, in the regime of nonlinear (i.e., nonOhmic) transport analogous to the VL in Fig. 1, in which the negative $R_{\mathrm{H}}$ arising from the vortex motion decreases towards zero as the doping approaches $x=1 / 8$ (Fig. 3b, d). The vanishing Hall response in $\mathrm{La}_{1.875} \mathrm{Ba}_{0.125} \mathrm{CuO}_{4}$ was indeed attributed ${ }^{37-39}$ to the presence of SC phase fluctuations and Cooper pairs that survive within the charge stripes after the inter-stripe SC phase coherence has been destroyed by $H$. Likewise, in $\mathrm{YBa}_{2} \mathrm{Cu}_{3} \mathrm{O}_{y}$ thin films near a disorder-tuned superconductor-insulator transition, $R_{\mathrm{H}}=0$ was found ${ }^{40}$ below the onset $T(\sim 80 \mathrm{~K})$ for SC fluctuations, at low fields up to $9 \mathrm{~T}$ and in the regime of strong positive MR consistent with the suppression of superconductivity. Both refs. ${ }^{37,40}$ reported $R_{\mathrm{H}}=0$ in an anomalous metallic regime with $\rho_{x x}(T \rightarrow 0) \neq 0$, similar to "failed superconductors" (or Bose metals) 34,35 . However, we note that in contrast, and unlike "failed superconductors" 34,35 , in $\mathrm{La}_{1.7} \mathrm{Eu}_{0.2} \mathrm{Sr}_{0.1} \mathrm{CuO}_{4}$ and $\mathrm{La}_{1.48} \mathrm{Nd}_{0.4} \mathrm{Sr}_{0.12} \mathrm{CuO}_{4}$, as in highly underdoped $\mathrm{La}_{2-x} \mathrm{Sr}_{x} \mathrm{CuO}_{4}$ (ref. $\left.{ }^{41}\right), \rho_{x x}(T \rightarrow 0)=0$ in the viscous $\mathrm{VL}^{3,4}$. In any case, we conclude that, in the entire $H<H_{\mathrm{c} 2}$ regime of these stripe-ordered cuprates, the Hall response is dominated by vortex physics. 

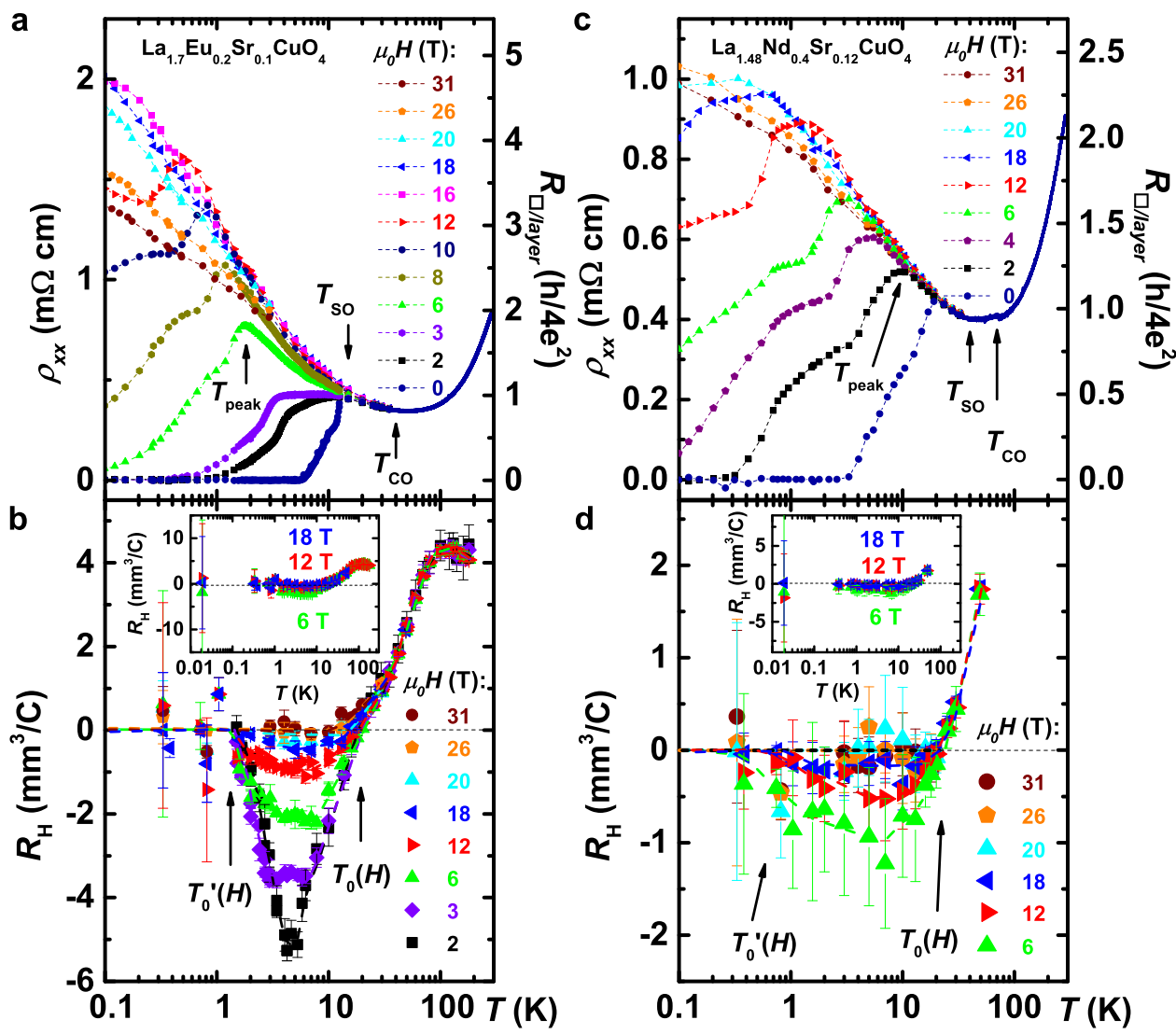

Fig. 3 Temperature dependence of the in-plane longitudinal resistivity $\rho_{\mathbf{x x}}$ and the Hall coefficient $\boldsymbol{R}_{\mathbf{H}}$ for various perpendicular $\boldsymbol{H}$. a, $\mathbf{b} \rho_{x x}$ and $R_{\mathrm{H}}$ respectively, for $\mathrm{La}_{1.7} \mathrm{Eu}_{0.2} \mathrm{Sr}_{0.1} \mathrm{CuO}_{4}$; the pseudogap temperature $T_{\mathrm{PG}} \sim 175 \mathrm{~K}$ (ref. ${ }^{55}$ ). c, d $\rho_{x x}$ and $R_{\mathrm{H}}$, respectively, for $\mathrm{La}_{1.48} \mathrm{Nd}_{0.4} \mathrm{Sr}_{0.12} \mathrm{CuO}_{4} ; T_{\mathrm{PG}} \sim 150 \mathrm{~K}$ (ref. ${ }^{55}$ ). The transition from the low-temperature orthorhombic to a low-temperature tetragonal structure occurs at $T_{\mathrm{d} 2} \sim 125 \mathrm{~K}_{\text {in }} \mathrm{La}_{1.7} \mathrm{Eu}_{0.2} \mathrm{Sr}_{0.1} \mathrm{CuO}_{4}$ and $T_{\mathrm{d} 2} \sim 70 \mathrm{~K}$ in $\mathrm{La}_{1.48} \mathrm{Nd}_{0.4} \mathrm{Sr}_{0.12} \mathrm{CuO}_{4}\left(\right.$ ref. $\left.^{3}\right)$. The data in $\mathbf{a}$ and $\mathbf{c}$ are from refs. ${ }^{3,4}$. At the highest fields, $\rho_{x x} \propto \ln (1 / T)$, as discussed in more detail elsewhere ${ }^{3}$. In both materials, $R_{\mathrm{H}}$ decreases upon cooling, and reaches zero at $T=T_{0}(H)$. For $H<H_{\mathrm{c} 2} \sim H_{\text {peak }}, R_{\mathrm{H}}$ becomes negative at even lower $T$, then goes through a minimum, and eventually reaches zero again at $T=T_{0}^{\prime}(H)$, as shown; $R_{\mathrm{H}}$ remains zero down to $0.019 \mathrm{~K}(\mathbf{b}$ and $\mathbf{d}$ insets $)$. For $H>H_{\mathrm{c} 2}, R_{\mathrm{H}}=0$ for all $H$ and $T<T_{0}(H)$. Similar to those in Fig. 2, error bars correspond to \pm 1 SD of the data points within each bin. All dashed lines guide the eye.

Transport in the $\boldsymbol{H}>\boldsymbol{H}_{\mathrm{c} 2}$ regime. The remaining, most intriguing question is the origin of $R_{\mathrm{H}}=0$ observed beyond the VL regime, at all $T<T_{0}$ and $H>H_{\text {peak }} \approx H_{\mathrm{c} 2}$ (blue areas in Fig. 1c, d). This anomalous normal state is also characterized ${ }^{3}$ by $\rho_{x x} \propto \ln (1 / T)$. In addition, here the out-of-plane resistivity has the same $T$-dependence ${ }^{4}, \rho_{c} \propto \ln (1 / T)$, implying that the transport mechanism is the same for both in-plane and $c$ directions. We discuss several potential scenarios for the origin of $R_{\mathrm{H}}=0$ in this regime.

\section{Discussion}

For $H>H_{\mathrm{c} 2}$, the first possibility to consider is whether there are any remnants of superconductivity, such as SC fluctuations that may no longer be detectable in the $\rho_{x x}$ measurement. In cuprates (ref. ${ }^{23}$ and refs. therein), as well as in conventional superconductors ${ }^{23,42}$, the effect of SC fluctuations on the Hall signal has been extensively studied in the high- $T$ normal state, at low fields and above $T_{c}^{0}$, within the conventional, weak-pairing fluctuation formalism built upon the Ginzburg-Landau (GL) theory of the BCS regime. The qualitative picture of SC fluctuations at low temperatures and high fields $(H>$ $\left.H_{\mathrm{c} 2}\right)$, however, drastically differs from the GL one ${ }^{23,43}$, but in either case, existing models predict nonzero $R_{\mathrm{H}}$ with particle-hole asymmetry terms ${ }^{23,43}$. Recently, a strong-pairing fluctuation theory that also incorporates pseudogap effects has been proposed ${ }^{24}$ for $R_{\mathrm{H}}$ in cuprates, but only for the low-field, $T>T_{c}^{0}$ regime. However, it does not describe the $H$-independence of the drop in $R_{\mathrm{H}}$ with decreasing $T$ observed for $T>T_{0}$ (Fig. 3b, d).

Extensive transport studies 3,4 , including those of the anisotropy ratio $\rho_{c} / \rho_{x x}$, have not found any observable signs of superconductivity, including the PDW, for $H>H_{\mathrm{c} 2}$. For example, here $\rho_{c} / \rho_{x x}$ no longer depends on a magnetic field, neither $H \| c$ nor $H \perp c$, and it reaches its high- $T$, normal-state value. As discussed elsewhere ${ }^{3}$, the value of $H_{\mathrm{c} 2} \approx H_{\text {peak }}$ is also consistent with the spectroscopic data for the closing of the SC gap in other cuprates. Although other experiments might be needed to definitively rule out the presence of any preformed pairs at $H>H_{\text {peak }}$, it appears far more likely that pairs cannot be responsible for $R_{\mathrm{H}}=0$ in the field-revealed normal state of $\mathrm{La}_{1.7} \mathrm{Eu}_{0.2} \mathrm{Sr}_{0.1} \mathrm{CuO}_{4}$ and $\mathrm{La}_{1.48} \mathrm{Nd}_{0.4} \mathrm{Sr}_{0.12} \mathrm{CuO}_{4}$, given also that $R_{\mathrm{H}}=0$ spans a $\sim 10 \mathrm{~T}$-wide range of fields in Fig. 1 . Therefore, models that rely on the existence of preformed pairs ${ }^{38,39}$, strong SC correlations such as those in "failed superconductors" 34,35 , or conventional Gaussian SC fluctuations ${ }^{23,43}$ do not seem relevant for the $H>H_{\mathrm{c} 2}$ regime. Hence, we consider other possible scenarios.

The drop of the positive $R_{\mathrm{H}}(T)$ to zero, observed at $T>T_{0}$, has been attributed $14,19,21,22$ to the Fermi surface reconstruction, implying the presence of both hole and electron pockets in the Fermi surface. Although this issue is not fully settled ${ }^{44}$, partly because of the disagreement with photoemission experiments, a similar drop of $R_{\mathrm{H}}(T)$ seen in $\mathrm{La}_{1.7} \mathrm{Eu}_{0.2} \mathrm{Sr}_{0.1} \mathrm{CuO}_{4}$ and $\mathrm{La}_{1.48} \mathrm{Nd}_{0.4} \mathrm{Sr}_{0.12} \mathrm{CuO}_{4}$ at $T>$ $T_{0}$ (Fig. $3 \mathrm{~b}, \mathrm{~d}$ ) suggests the possibility that the same mechanism might be responsible for the normal-state behavior of $R_{\mathrm{H}}$ at $T>T_{0}$ in 
these stripe-ordered cuprates, and even in their $T<T_{0}$, high-field regime, which is the focus of our study. We note, however, that there is no consensus on how the Fermi surface is affected by the presence of spin stripes, including in $\mathrm{La}_{2-x-y} \mathrm{Sr}_{x}(\mathrm{Nd}, \mathrm{Eu})_{y} \mathrm{CuO}_{4}$ compounds near $x=1 / 8$. Therefore, without additional input from other techniques, any multiband model with a sufficient number of fitting parameters could reproduce our result that, in the high-field normal state, $R_{\mathrm{H}}=0$ within our measurement resolution (1 SD), $\Delta R_{\mathrm{H}} \sim$ $0.05 \mathrm{~mm}^{3} / \mathrm{C}$ (or standard error $\sim 0.01 \mathrm{~mm}^{3} / \mathrm{C}$; see Methods). We note that the latter is comparable to, if not better than, $\Delta R_{\mathrm{H}}$ in other similar studies (see Methods for a detailed discussion of the experimental resolution and unique measurement challenges). However, our results, in fact, place stringent constraints on any realistic models for the Hall effect in this regime: $R_{\mathrm{H}}<0.05 \mathrm{~mm}^{3} / \mathrm{C}$, but this condition also needs to be satisfied over a wide range of $H$ and $T$ for two different materials and doping levels (Fig. 1). In a multiband picture, this would require that a subtle balance, or a near-cancellation, of contributions from hole and electron pockets is maintained over a huge range of parameters $T$ and $H$, as well as change in $x$ and the rare-earth composition $y$. Therefore, a multiband picture seems unlikely considering the robustness of our results.

Since $d \rho_{x x} / d T<0$ in the normal state, one could speculate whether $R_{\mathrm{H}}$ vanishes (i.e. $\rho_{y x}=0$, or conductivity $\sigma_{x y}=0$ ) because of some kind of localization. Strong, exponential localization does not describe the data because the $T$-dependence of the resistivity is very weak, it becomes even weaker with increasing $H$, and at the same time, the absolute value of $\rho_{x x}$ remains relatively low and comparable to that at $T>T_{0}$ (Fig. 3a, c). Similarly, as the system goes from the VL to the normal state with $H$ at a fixed, relatively high $T<T_{0}$, the $H$-dependence of $\rho_{x x}$ is negligible ${ }^{3}$ (e.g., at $\sim 4 \mathrm{~K}$ in Fig. 3a), while $R_{\mathrm{H}}$ changes qualitatively from a finite negative value to zero (Fig. $3 \mathrm{~b}$ ). Our results for $R_{\mathrm{H}}$ are indeed the opposite of those in lightly-doped ${ }^{17}$, i.e. insulating cuprates with a diverging $\rho_{x x}(T \rightarrow 0)$, or in highly underdoped ${ }^{16}$ cuprates, both of which seem to show a diverging $R_{\mathrm{H}}$ at low $T$. If $n=n_{\mathrm{H}}=1 /\left(e R_{\mathrm{H}}\right)$ holds, this is indeed consistent with a depletion of carriers, whereas in our case it would indicate a diverging number of carriers. Likewise, weak localization in $2 \mathrm{D}$ is not consistent with the data, since the same $\ln (1 / T)$ behavior is observed also along the $c$ axis, just like in underdoped $\mathrm{La}_{2-x} \mathrm{Sr}_{x} \mathrm{CuO}_{4}$ (ref. ${ }^{6}$ ). While weak localization does not produce a correction to the classical $R_{\mathrm{H}}$ value, electron-electron interactions in weakly disordered $2 \mathrm{D}$ metals give rise ${ }^{45}$ to logarithmic corrections to $\rho_{x x}$ and $R_{\mathrm{H}}$, which are related such that $\delta R_{\mathrm{H}} / R_{\mathrm{H}}=2\left(\delta \rho_{x x} / \rho_{x x}\right)$. However, just like in $\mathrm{La}_{2-x} \mathrm{Sr}_{x} \mathrm{CuO}_{4}$ (ref. $\left.{ }^{6}\right)$, this is not consistent with our observation ${ }^{3}$ of a large $\ln (1 / T)$ term in $\rho_{x x}$, and it does not describe the vanishing $R_{\mathrm{H}}$. Hence, standard localization mechanisms cannot explain $R_{\mathrm{H}}=0$ observed over a wide range of $T<T_{0}$ and $H>H_{\mathrm{c} 2}$.

On the other hand, a confinement of carriers within 1D charge stripes, associated with the suppression of the cyclotron motion with increasing $H$, was proposed to understand the drop of the positive $R_{\mathrm{H}}(T)$ towards zero observed ${ }^{28}$ in $\mathrm{La}_{2-x-y} \mathrm{Nd}_{y} \mathrm{Sr}_{x} \mathrm{CuO}_{4}$ near $x=1 / 8$ at low $H=5 \mathrm{~T}$ and high $T$, i.e. $T>T_{0}$ in Fig. $3 \mathrm{~b}, \mathrm{~d}$. Although, in contrast, our central result is $R_{\mathrm{H}}=0$ in the highfield $\left(H>H_{\mathrm{c} 2}\right), T<T_{0}$ regime, models based on the quasi-1D picture seem to be a plausible description of stripe-ordered cuprates also when the applied $H$ suppresses the interstripe Josephson coupling. One such model, for example, predicts ${ }^{46}$, both in the presence and the absence of a spin gap, a non-Fermiliquid smectic metal phase, in which the transport across the stripes is incoherent, whereas it is coherent inside each stripe. Importantly, a smectic metal has an approximate particle-hole symmetry ${ }^{46}$ for $x<1 / 8$, which implies $\rho_{y x} \approx 0$, as observed in our experiment. Incidentally, the same model had been proposed as the origin of the drop of $R_{\mathrm{H}}$ in the early studies ${ }^{47}$ of $\mathrm{YBa}_{2} \mathrm{Cu}_{3} \mathrm{O}_{y}$ at $T>T_{0}$. Other, more general scenarios include holographic models for doped Mott insulators ${ }^{48}$, which also feature emergent particlehole symmetry ${ }^{49}$.

Our study of the Hall effect across the entire in-plane $T-H$ phase diagram has clarified and further confirmed that the origin of $R_{\mathrm{H}}=0$ reported in earlier studies $28,30,31,37$ of stripe-ordered cuprates is associated with the presence of SC fluctuations. In contrast, our central result is that, at much higher fields, such that $H>H_{\mathrm{c} 2}$, the field-revealed normal state of $\mathrm{La}_{2-x-y} \mathrm{Sr}_{x}(\mathrm{Nd}$, $\mathrm{Eu})_{y} \mathrm{CuO}_{4}$ cuprates with static spin and charge stripes is characterized by a zero, i.e. immeasurably small, Hall coefficient. Indeed, since the vanishing of $R_{\mathrm{H}}$ is pronounced over a wider range of $H$ and $T$ for $x=0.12$ (Fig. $1 \mathrm{~b}, \mathrm{~d}$ ) than for $x=0.10$ (Fig. 1a, c), this strongly suggests that $R_{\mathrm{H}} \approx 0$ is crucially related to the presence of static stripe order. Further insight into this issue might come from other experiments at high fields, such as optical conductivity, Raman scattering, and thermal transport, to determine whether $R_{\mathrm{H}} \approx 0$ results from a fortuitous nearcancellation of contributions from multiple bands or it signals an approximate particle-hole symmetry, as expected for a smectic metal in a stripe-ordered cuprate ${ }^{46}$ and in more general models of correlated matter ${ }^{48,49}$.

\section{Methods}

Samples. Several single crystal samples of $\mathrm{La}_{1.8-x} \mathrm{Eu}_{0.2} \mathrm{Sr}_{x} \mathrm{CuO}_{4}$ with a nominal $x=0.10$ and $\mathrm{La}_{1.6-x} \mathrm{Nd}_{0.4} \mathrm{Sr}_{x} \mathrm{CuO}_{4}$ with a nominal $x=0.12$ were grown by the traveling-solvent floating-zone technique ${ }^{50}$. The high quality of the crystals was confirmed by several techniques, as discussed in detail elsewhere ${ }^{3,4}$. The samples were shaped as rectangular bars suitable for direct measurements of the longitudinal and transverse (Hall) resistance, $R_{x x}$ and $R_{y x}$, respectively. Detailed measurements of $R_{x x}$ and $R_{y x}$ were performed on $\mathrm{La}_{1.7} \mathrm{Eu}_{0.2} \mathrm{Sr}_{0.1} \mathrm{CuO}_{4}$ sample "B" with dimensions $3.06 \times 0.53 \times 0.37 \mathrm{~mm}^{3}(a \times b \times c$, i.e. length $\times$ width $\times$ thickness $)$ and a $\mathrm{La}_{1.48} \mathrm{Nd}_{0.4} \mathrm{Sr}_{0.12} \mathrm{CuO}_{4}$ crystal with dimensions $3.82 \times 1.19 \times 0.49 \mathrm{~mm}^{3}$. The same two samples were also studied previously ${ }^{3,4}$. After $\sim 3$ years, the low- $T$ properties of the $\mathrm{La}_{1.7} \mathrm{Eu}_{0.2} \mathrm{Sr}_{0.1} \mathrm{CuO}_{4}$ sample "B" changed, which was attributed to a small change (increase) in the effective doping, but its phases remained qualitatively the same ${ }^{4}$. We repeated the Hall measurements after the sample had changed, and obtained the same results.

Gold contacts were evaporated on polished crystal surfaces, and annealed in air at $700{ }^{\circ} \mathrm{C}$. The current contacts were made by covering the whole area of the two opposing sides with gold to ensure uniform current flow, and the voltage contacts were made narrow to minimize the uncertainty in the absolute values of the resistance. Multiple voltage contacts on opposite sides of the crystals were prepared, and the results did not depend on the position of the contacts. Gold leads $(\approx 25 \mu \mathrm{m}$ thick) were attached to the samples using the Dupont 6838 silver paste, followed by the heat treatment at $450{ }^{\circ} \mathrm{C}$ in the flow of oxygen for $15 \mathrm{~min}$. The resulting contact resistances were less than $0.1 \Omega$ for $\mathrm{La}_{1.7} \mathrm{Eu}_{0.2} \mathrm{Sr}_{0.1} \mathrm{CuO}_{4}(0.5 \Omega$ for $\mathrm{La}_{1.48} \mathrm{Nd}_{0.4} \mathrm{Sr}_{0.12} \mathrm{CuO}_{4}$ ) at room temperature. Meanwhile, we found no change in the superconducting properties of the samples before and after the annealing.

Measurements. The standard ac lock-in techniques $(\sim 13 \mathrm{~Hz})$ were used for measurements of $R_{x x}$ and $R_{y x}$ with the magnetic field parallel and anti-parallel to the $c$ axis. The Hall resistance was determined from the transverse voltage by extracting the component antisymmetric in the magnetic field. The Hall coefficient $R_{H}=R_{y x} d / H=\rho_{y x} / H$, where $d$ is the sample thickness. The $\rho_{x x}$ data measured simultaneously with $\rho_{y x}$ agree well with the previously reported results of magnetoresistance measurements 3,4 . The resistance per square per $\mathrm{CuO}_{2}$ layer $R_{\square}$ /layer $=\rho_{x x} / l$, where $l=6.6 \AA$ is the thickness of each layer.

Depending on the temperature, the excitation current (density) of $10 \mu \mathrm{A}$ to $316 \mu \mathrm{A}\left(\sim 5 \times 10^{-3} \mathrm{~A} \mathrm{~cm}^{-2}\right.$ to $\sim 1.6 \times 10^{-1} \mathrm{~A} \mathrm{~cm}^{-2}$ for $\mathrm{La}_{1.7} \mathrm{Eu}_{0.2} \mathrm{Sr}_{0.1} \mathrm{CuO}_{4}$, and $\sim 2 \times 10^{-3} \mathrm{~A} \mathrm{~cm}^{-2}$ to $\sim 6.3 \times 10^{-2} \mathrm{~A} \mathrm{~cm}^{-2}$ for $\mathrm{La}_{1.48} \mathrm{Nd}_{0.4} \mathrm{Sr}_{0.12} \mathrm{CuO}_{4}$ ) was used: $10 \mu \mathrm{A}$ for $0.019 \mathrm{~K}$ (Supplementary Fig. 1d); $100 \mu \mathrm{A}$ for all measurements in fields up to $12 \mathrm{~T}$ (Supplementary Fig. 1a), and for the $0.3 \mathrm{~K}$ data in Supplementary Figs. 2 and 3; $316 \mu \mathrm{A}$ for all other measurements. These excitation currents were low enough to avoid Joule heating ${ }^{3}$. Traces with different excitation currents were also compared to ensure that the reported results are in the linear response regime. A $1 \mathrm{k} \Omega$ resistor in series with a $\pi$ filter $[5 \mathrm{~dB}(60 \mathrm{~dB})$ noise reduction at $10 \mathrm{MHz}$ $(1 \mathrm{GHz})]$ was placed in each wire at the room temperature end of the cryostat to reduce the noise and heating by radiation in all measurements.

Several different cryostats at the National High Magnetic Field Laboratory were used, including a dilution refrigerator $(0.016 \mathrm{~K} \leqslant \mathrm{~T} \leqslant 0.7 \mathrm{~K})$ and a ${ }^{3} \mathrm{He}$ system $(0.3 \mathrm{~K} \leqslant T \leqslant 35 \mathrm{~K})$ in superconducting magnets ( $H$ up to $18 \mathrm{~T}$ ), using $0.1-0.2 \mathrm{~T} /$ min sweep rates, and a ${ }^{3} \mathrm{He}$ system $(0.3 \mathrm{~K} \leqslant T \leqslant 20 \mathrm{~K})$ in a $31 \mathrm{~T}$ resistive magnet, using 1-2 $\mathrm{T} / \mathrm{min}$ sweep rates. Some of the measurements were performed in a variable-temperature insert $(1.7 \mathrm{~K} \leq T \leq 200 \mathrm{~K})$ with a $12 \mathrm{~T}$ superconducting magnet. The fields were swept at constant temperatures, and the sweep rates were 
low enough to avoid eddy current heating of the samples. The results obtained in different magnets and cryostats agree well.

Experimental resolution of Hall effect measurements. Unlike other cuprates such as YBCO, in which $\rho_{x x}$ and $\rho_{y x}$ are comparable at low $T$ and high $H$ (e.g. ref. ${ }^{51}$ ), $\rho_{y x}$ is orders of magnitude smaller than $\rho_{x x}$ in $\mathrm{La}_{1.7} \mathrm{Eu}_{0.2} \mathrm{Sr}_{0.1} \mathrm{CuO}_{4}$ and $\mathrm{La}_{1.48} \mathrm{Nd}_{0.4} \mathrm{Sr}_{0.12} \mathrm{CuO}_{4}$, even in the high- $T$ normal state. For example, as seen from Supplementary Fig. 1a for $\mathrm{La}_{17} \mathrm{Eu}_{0.2} \mathrm{Sr}_{0.1} \mathrm{CuO}_{4}$ at $T=180 \mathrm{~K}$ and $H=12 \mathrm{~T}, \rho_{y x}=$ $R_{y x} d \sim 0.005 \mathrm{~m} \Omega \mathrm{cm}$, while $\rho_{x x} \gtrsim 0.5 \mathrm{~m} \Omega \mathrm{cm}$ (see Fig. 3 for $H=0$, but at $T>15 \mathrm{~K}$, the magnetoresistance is very weak ${ }^{3,52}$ ). At low $T, \rho_{y x}$ is drastically suppressed even further (Supplementary Fig. 1), and the ratio $\rho_{y x} / \rho_{x x}$ becomes even greater. This observation is significant in itself as discussed in the main text, but it also presents certain experimental challenges.

In a standard Hall measurement, any contribution of $\rho_{x x}$, which results from a slight misalignment of voltage contacts, is removed and $\rho_{y x}$ is isolated by antisymmetrization of the transverse voltage drops measured with a field both parallel and antiparallel to the $c$ axis. However, a perfect cancellation of the $\rho_{x x}$ contribution can only be achieved if the two measurements in opposite field directions are conducted at exactly the same $T$ (and other experimental conditions). Otherwise, $\rho_{x x}$ can contaminate the Hall resistivity even after the conventional antisymmetrization procedure, especially if $\rho_{x x}$ is much larger than $\rho_{y x}$ and it has a strong temperature dependence as in $\mathrm{La}_{1.7} \mathrm{Eu}_{0.2} \mathrm{Sr}_{0.1} \mathrm{CuO}_{4}$ and $\mathrm{La}_{1.48} \mathrm{Nd}_{0.4} \mathrm{Sr}_{0.12} \mathrm{CuO}_{4}$. Therefore, careful temperature control during the experiment and meticulous data analysis afterwards are key to our Hall measurements on these two systems.

With the single-shot ${ }^{3} \mathrm{He}$ cryostat, the temperature control below $1.6 \mathrm{~K}$ is usually complicated by the evaporation of the ${ }^{3} \mathrm{He}$ liquid, which induces a slow $\mathrm{T}$ drift with time. To minimize its impact, we measured the traces with opposite fields in back-to-back experiments and did not consider the data when the $T$ drift was too large. The maximum $T$ drift between the two traces is typically $\sim 10-20 \mathrm{mK}$ for $T<1.6 \mathrm{~K}$.

To ensure the accuracy of our results, we have also repeated Hall measurements on $\mathrm{La}_{1.7} \mathrm{Eu}_{0.2} \mathrm{Sr}_{0.1} \mathrm{CuO}_{4}$ at $0.71 \mathrm{~K}$ more than 10 times, by recondensing the ${ }^{3} \mathrm{He}$ liquid and resetting the temperature for each positive and negative field sweep. This ensures that, even if $T$ drifts due to ${ }^{3} \mathrm{He}$ evaporation, the amount of the drift would be the same in the two traces. We carefully compared the field dependence of the Cernox ${ }^{\oplus}$ thermometer reading, $T_{r}$, for each positive and negative field sweep, a typical example of which is shown in Supplementary Fig. 4a inset. We note that the Cernox sensor is not calibrated in the field, and thus the increase of $T_{r}$ only reflects the magnetoresistance of the sensor, while the sample temperature (controlled by the sorb) is unchanged. As shown in the Supplementary Fig. 4a inset, the temperature is the same (within $1 \mathrm{mK}$ ) during the entire positive and negative field sweeps.

To determine the uncertainty of the Hall coefficient measurement results, we divide the $R_{\mathrm{yx}}(H)$ and $R_{\mathrm{H}}(H)$ data into bins (typical size is $1 \mathrm{~T}$; see Supplementary Figs. 2 and 3), and calculate the mean and the standard deviation (SD) within each bin. Therefore, the error bars in Figs. 2 and 3, and Supplementary Figs. 1, 2, 3, 4, and 6 , all correspond to $\pm 1 \mathrm{SD}$ of the data points within each bin. To reduce the $\mathrm{SD}$ even further, we averaged over five sets of measurements at $0.71 \mathrm{~K}$ to reduce the experimental error bar (i.e. $1 \mathrm{SD}$ ) from $\Delta R_{\mathrm{H}} \sim 0.2 \mathrm{~mm}^{3} / \mathrm{C}$ to $\Delta R_{\mathrm{H}} \sim 0.05 \mathrm{~mm}^{3} / \mathrm{C}$ (Supplementary Fig. 4b). This is comparable to, if not better than, $\Delta R_{\mathrm{H}}$ in other studies of the Hall effect on cuprates ${ }^{14}$, including those in which zero Hall coefficient (induced by superconductivity, not in the normal state) was found $27,30,37,40$, as well as on other systems, such as iron-based superconductors ${ }^{53}$ We emphasize again that the experimental error for $\rho_{y x}$ (and $R_{\mathrm{H}}$ ) is dominated by the imperfect cancellation of the contribution from the $T$-dependent longitudinal resistivity $\rho_{x x}$, which is inevitably much larger than the (nearly) zero transverse contribution. At $T=0.71 \mathrm{~K}$, where we achieved almost perfect temperature control (to within $1 \mathrm{mK}$ ) and thus the maximum cancellation of the longitudinal resistivity contribution (Supplementary Fig. 4a), we also determined, using standard error analysis, the $\sim 95 \%$ confidence intervals for $R_{\mathrm{H}}$, e.g., $-0.008 \pm 0.020 \mathrm{~mm}^{3} / \mathrm{C}$ at $17 \mathrm{~T}$. This further confirms our conclusion that the Hall coefficient (and Hall resistivity, see Supplementary Fig. 1e inset) remains zero in the high-field normal state, i.e., above the upper critical field $H_{\text {peak. }}$.

In principle, the error bar in the $\rho_{y x}$ measurement can also be reduced by increasing the excitation current density or, equivalently, by reducing the sample thickness or width for a fixed current. However, the applied current density still needs to remain below the limit above which Joule heating is induced. The effects of excitation currents have been studied thoroughly ${ }^{3}$, so that here we have used the highest excitation current density possible without inducing Joule heating. Therefore, reducing the sample thickness, for example, would not help to decrease the error bar further, because a smaller excitation current would also need to be used.

\section{Data availability}

The data that support the findings of this study are available within the paper and the Supplementary Information. Additional data related to this paper may be requested from the authors.
Received: 2 October 2020; Accepted: 25 May 2021; Published online: 17 June 2021

\section{References}

1. Keimer, B., Kivelson, S. A., Norman, M. R., Uchida, S. \& Zaanen, J. From quantum matter to high-temperature superconductivity in copper oxides. Nature 518, 179-186 (2015).

2. Fradkin, E., Kivelson, S. A. \& Tranquada, J. M. Colloquium: theory of intertwined orders in high temperature superconductors. Rev. Mod. Phys. 87, 561-563 (2015)

3. Shi, Z., Baity, P. G., Sasagawa, T. \& Popović, D. Vortex phase diagram and the normal state of cuprates with charge and spin orders. Sci. Adv. 6, eaay8946 (2020)

4. Shi, Z., Baity, P. G., Terzic, J., Sasagawa, T. \& Popović, D. Pair density wave at high magnetic fields in cuprates with charge and spin orders. Nat. Commun. 11, 3323 (2020).

5. Agterberg, D. F. et al. The physics of pair density waves: Cuprate superconductors and beyond. Annu. Rev. Condens. Matter Phys. 11, 231-270 (2020).

6. Ando, Y., Boebinger, G. S., Passner, A., Kimura, T. \& Kishio, K. Logarithmic divergence of both in-plane and out-of-plane normal-state resistivities of superconducting $\mathrm{La}_{2-x} \mathrm{Sr}_{x} \mathrm{CuO}_{4}$ in the zero-temperature limit. Phys. Rev. Lett. 75, 4662-4665 (1995).

7. Ono, S. et al. Metal-to-insulator crossover in the low-temperature normal state of $\mathrm{Bi}_{2} \mathrm{Sr}_{2-x} \mathrm{La}_{x} \mathrm{CuO}_{6+\delta}$. Phys. Rev. Lett. 85, 638-641 (2000).

8. Jacobsen, $\mathrm{H}$. et al. Neutron scattering study of spin ordering and stripe pinning in superconducting $\mathrm{La}_{1.93} \mathrm{Sr}_{0.07} \mathrm{CuO}_{4}$. Phys. Rev. B 92, 174525 (2015).

9. Boebinger, G. S. et al. Insulator-to-metal crossover in the normal state of $\mathrm{La}_{2}$ ${ }_{-x} \mathrm{Sr}_{x} \mathrm{CuO}_{4}$ near optimum doping. Phys. Rev. Lett. 77, 5417-5420 (1996)

10. Fournier, P. et al. Insulator-metal crossover near optimal doping in $\mathrm{Pr}_{2}$ ${ }_{-x} \mathrm{Ce}_{x} \mathrm{CuO}_{4}$. Phys. Rev. Lett. 81, 4720-4723 (1998).

11. Sun, X. F., Segawa, K. \& Ando, Y. Low-temperature nodal-quasiparticle transport in lightly doped $\mathrm{YBa}_{2} \mathrm{Cu}_{3} \mathrm{O}_{y}$ near the edge of the superconducting doping regime. Phys. Rev. B 72, 100502 (2005).

12. Rullier-Albenque, F., Alloul, H., Balakirev, F. \& Proust, C. Disorder, metalinsulator crossover and phase diagram in high- $T_{c}$ cuprates. EPL 81, 37008 (2008).

13. Zhou, X. et al. Logarithmic upturn in low-temperature electronic transport as a signature of $d$-wave order in cuprate superconductors. Phys. Rev. Lett. 121, 267004 (2018).

14. LeBoeuf, D. et al. Electron pockets in the Fermi surface of hole-doped high- $T_{c}$ superconductors. Nature 450, 533-536 (2007)

15. Doiron-Leyraud, N. et al. Hall, Seebeck, and Nernst coefficients of underdoped $\mathrm{HgBa}_{2} \mathrm{CuO}_{4+\delta}$ : Fermi-surface reconstruction in an archetypal cuprate superconductor. Phys. Rev. X 3, 021019 (2013).

16. Balakirev, F. F. et al. Signature of optimal doping in Hall-effect measurements on a high-temperature superconductor. Nature 424, 912-915 (2003).

17. Ando, Y., Kurita, Y., Komiya, S., Ono, S. \& Segawa, K. Evolution of the Hall coefficient and the peculiar electronic structure of the cuprate superconductors. Phys. Rev. Lett. 92, 197001 (2004)

18. Badoux, S. et al. Change of carrier density at the pseudogap critical point of a cuprate superconductor. Nature 531, 210-214 (2016).

19. Badoux, S. et al. Critical doping for the onset of Fermi-surface reconstruction by charge-density-wave order in the cuprate superconductor $\mathrm{La}_{2-x} \mathrm{Sr}_{x} \mathrm{CuO}_{4}$. Phys. Rev. X 6, 021004 (2016)

20. Comin, R. \& Damascelli, A. Resonant X-ray scattering studies of charge order in cuprates. Annu. Rev. Condens. Matter Phys. 7, 369-405 (2016).

21. Taillefer, L. Fermi surface reconstruction in high- $T_{c}$ superconductors. J. Phys.: Condens. Matter 21, 164212 (2009).

22. LeBoeuf, D. et al. Lifshitz critical point in the cuprate superconductor $\mathrm{YBa}_{2} \mathrm{Cu}_{3} \mathrm{O}_{y}$. Phys. Rev. B 83, 054506 (2011).

23. Varlamov, A. A., Galda, A. \& Glatz, A. Fluctuation spectroscopy: from Rayleigh-Jeans waves to Abrikosov vortex clusters. Rev. Mod. Phys. 90, 015009 (2018).

24. Boyack, R., Wang, W., Chen, Q. \& Levin, K. Combined effects of pairing fluctuations and a pseudogap in the cuprate Hall coefficient. Phys. Rev. B 99, 134504 (2019).

25. Balakirev, F. F. et al. Quantum phase transition in the magnetic-field-induced normal state of optimum-doped high- $T_{c}$ cuprate superconductors at low temperatures. Phys. Rev. Lett. 102, 017004 (2009).

26. Putzke, C. et al. Reduced Hall carrier density in the overdoped strange metal regime of cuprate superconductors. Nat. Phys. https://doi.org/10.1038/s41567021-01197-0 (2021) 
27. Stegen, Z. et al. Evolution of superconducting correlations within magneticfield-decoupled $\mathrm{La}_{2-x} \mathrm{Ba}_{x} \mathrm{CuO}_{4}(x=0.095)$. Phys. Rev. B 87, 064509 (2013).

28. Noda, T., Eisaki, H. \& Uchida, S.-I. Evidence for one-dimensional charge transport in $\mathrm{La}_{2-x-y} \mathrm{Nd}_{y} \mathrm{Sr}_{x} \mathrm{CuO}_{4}$. Science 286, 265-268 (1999).

29. Adachi, T., Noji, T. \& Koike, Y. Crystal growth, transport properties, and crystal structure of the single-crystal $\mathrm{La}_{2-x} \mathrm{Ba}_{x} \mathrm{CuO}_{4}(x=0.11)$. Phys. Rev. $B$ 64, 144524 (2001).

30. Adachi, T., Kitajima, N. \& Koike, Y. Hall coefficient in the ground state of stripe-ordered $\mathrm{La}_{2-x} \mathrm{Ba}_{x} \mathrm{CuO}_{4}$ single crystals. Phys. Rev. B 83, 060506 (2011).

31. Xie, L., Ding, J. F., Guo, R. R., Sun, X. F. \& Li, X. G. Interplay between charge stripes and sign reversals of Hall and Seebeck effects in stripe-ordered $\mathrm{La}_{1.6-x} \mathrm{Nd}_{0.4} \mathrm{Sr}_{x} \mathrm{CuO}_{4}$ superconductors. J. Phys. Condens. Matter 23, 365702 (2011).

32. Vinokur, V. M., Geshkenbein, V. B., Feigel'man, M. V. \& Blatter, G. Scaling of the Hall resistivity in high- $T_{c}$ superconductors. Phys. Rev. Lett. 71, 1242-1245 (1993).

33. Delacrétaz, L. V. \& Hartnoll, S. A. Theory of the supercyclotron resonance and Hall response in anomalous two-dimensional metals. Phys. Rev. B 97, 220506 (R) (2018)

34. Breznay, N. P. \& Kapitulnik, A. Particle-hole symmetry reveals failed superconductivity in the metallic phase of two-dimensional superconducting films. Sci. Adv. 3, e1700612 (2017).

35. Kapitulnik, A., Kivelson, S. A. \& Spivak, B. Colloquium: anomalous metals: failed superconductors. Rev. Mod. Phys. 91, 011002 (2019).

36. Chen, Z. et al. Universal behavior of the bosonic metallic ground state in a two-dimensional superconductor. npj Quantum Mater. 6, 15 (2021).

37. Li, Y. et al. Tuning from failed superconductor to failed insulator with magnetic field. Sci. Adv. 5, eaav7686 (2019).

38. Tsvelik, A. M. Superconductor-metal transition in odd-frequency-paired superconductor in a magnetic field. PNAS 116, 12729-12732 (2019).

39. Ren, T. \& Tsvelik, A. M. How magnetic field can transform a superconductor into a Bose metal. N. J. Phys. 22, 103021 (2020).

40. Yang, C. et al. Intermediate bosonic metallic state in the superconductorinsulator transition. Science 366, 1505-1509 (2019).

41. Shi, X., Lin, P. V., Sasagawa, T., Dobrosavljević, V. \& Popović, D. Two-stage magnetic-field-tuned superconductor-insulator transition in underdoped $\mathrm{La}_{2-x} \mathrm{Sr}_{x} \mathrm{CuO}_{4}$. Nat. Phys. 10, 437-443 (2014).

42. Destraz, D., Ilin, K., Siegel, M., Schilling, A. \& Chang, J. Superconducting fluctuations in a thin NbN film probed by the Hall effect. Phys. Rev. B 95, 224501 (2017).

43. Michaeli, K., Tikhonov, K. S. \& Finkel'stein, A. M. Hall effect in superconducting films. Phys. Rev. B 86, 014515 (2012).

44. Sebastian, S. E. \& Proust, C. Quantum oscillations in hole-doped cuprates. Annu. Rev. Condens. Matter Phys. 6, 411-430 (2015).

45. Lee, P. A. \& Ramakrishnan, T. V. Disordered electronic systems. Rev. Mod. Phys. 57, 287-337 (1985).

46. Emery, V. J., Fradkin, E., Kivelson, S. A. \& Lubensky, T. C. Quantum theory of the smectic metal state in stripe phases. Phys. Rev. Lett. 85, 2160-2163 (2000).

47. Ando, Y. \& Segawa, K. Magnetotransport properties of untwinned $\mathrm{YBa}_{2} \mathrm{Cu}_{3} \mathrm{O}_{y}$ single crystals: Novel 60-K-phase anomalies in the charge transport. J. Phys. Chem. Solids 63, 2253-2257 (2002).

48. Andrade, T., Krikun, A., Schalm, K. \& Zaanen, J. Doping the holographic Mott insulator. Nat. Phys. 14, 1049-1055 (2018).

49. Blake, M. \& Donos, A. Quantum critical transport and the Hall angle in holographic models. Phys. Rev. Lett. 114, 021601 (2015).

50. Takeshita, N., Sasagawa, T., Sugioka, T., Tokura, Y. \& Takagi, H. Gigantic anisotropic uniaxial pressure effect on superconductivity within the $\mathrm{CuO}_{2}$ plane of $\mathrm{La}_{1.64} \mathrm{Eu}_{0.2} \mathrm{Sr}_{0.16} \mathrm{CuO}_{4}$ : strain control of stripe criticality. J. Phys. Soc. Jpn. 73, 1123-1126 (2004).

51. Grissonnanche, G. et al. Wiedemann-Franz law in the underdoped cuprate superconductor $\mathrm{YBa}_{2} \mathrm{Cu}_{3} \mathrm{O}_{y}$. Phys. Rev. B 93, 064513 (2016).
52. Chang, J. et al. Decrease of upper critical field with underdoping in cuprate superconductors. Nat. Phys. 8, 751-756 (2012).

53. Lin, H. et al. Multiband superconductivity and large anisotropy in FeS crystals. Phys. Rev. B 93, 144505 (2016).

54. Fink, J. et al. Phase diagram of charge order in $\mathrm{La}_{1.8-x} \mathrm{Eu}_{0.2} \mathrm{Sr}_{x} \mathrm{CuO}_{4}$ from resonant soft x-ray diffraction. Phys. Rev. B 83, 092503 (2011).

55. Cyr-Choinière, O. et al. Pseudogap temperature $T^{*}$ of cuprate superconductors from the Nernst effect. Phys. Rev. B 97, 064502 (2018).

56. Tranquada, J. M. et al. Neutron-scattering study of stripe-phase order of holes and spins in $\mathrm{La}_{1.48} \mathrm{Nd}_{0.4} \mathrm{Sr}_{0.12} \mathrm{CuO}_{4}$. Phys. Rev. B 54, 7489-7499 (1996).

\section{Acknowledgements}

We thank G. Saraswat for experimental assistance, and J. M. Tranquada, K. Yang, J. Zaanen for helpful discussions. This work was supported by NSF Grants Nos. DMR1307075 and DMR-1707785, and the National High Magnetic Field Laboratory (NHMFL) through the NSF Cooperative Agreements Nos. DMR-1157490, DMR1644779, and the State of Florida. Z.S. acknowledges support by the Priority Academic Program Development of Jiangsu Higher Education Institutions (PAPD). Z.S. is also grateful for the support from Jiangsu Key Laboratory of Thin Films and Jiangsu Key Lab of Advanced Optical Manufacturing Technologies.

\section{Author contributions}

Single crystals were grown and prepared by T.S.; Z.S., P.G.B., J.T. and B.K.P. performed the measurements; Z.S. analyzed the data; Z.S. and D.P. wrote the manuscript, with input from all authors; D.P. supervised the project.

\section{Competing interests}

The authors declare no competing interests.

\section{Additional information}

Supplementary information The online version contains supplementary material available at https://doi.org/10.1038/s41467-021-24000-3.

Correspondence and requests for materials should be addressed to D.Pć.

Peer review information Nature Communications thanks the anonymous reviewers for their contribution to the peer review of this work. Peer reviewer reports are available.

Reprints and permission information is available at http://www.nature.com/reprints

Publisher's note Springer Nature remains neutral with regard to jurisdictional claims in published maps and institutional affiliations.

(c) (i) Open Access This article is licensed under a Creative Commons Attribution 4.0 International License, which permits use, sharing, adaptation, distribution and reproduction in any medium or format, as long as you give appropriate credit to the original author(s) and the source, provide a link to the Creative Commons license, and indicate if changes were made. The images or other third party material in this article are included in the article's Creative Commons license, unless indicated otherwise in a credit line to the material. If material is not included in the article's Creative Commons license and your intended use is not permitted by statutory regulation or exceeds the permitted use, you will need to obtain permission directly from the copyright holder. To view a copy of this license, visit http://creativecommons.org/ licenses/by/4.0/.

(C) The Author(s) 2021 